\section{Breast operative technique for single-stage reconstruction after conservative skin sparing and nipple sparing mastectomies: a preliminary study}

\author{
Egidio Riggio, Joseph Ottolenghi, \\ Maurizio B. Nava
}

Plastic and Reconstructive Surgery Unit, Fondazione IRCCS Istituto Nazionale dei Tumori, Milano, Italy

\section{Abstract}

The Authors present a novel technique of immediate breast reconstruction with definite implants after mastectomy conserving the nipple-areola complex and, less frequently, in skin-sparing mastectomy. The increase of indications for both oncologic and prophylactic nipple-sparing mastectomy has induced the research for a single-stage technique that could replace the two-stage reconstruction with expanders and/or autogenous reconstructions with flaps. The new techniques introduce modifications of the pocket coverage for the implants occurring in two ways: i) autologous adaptation of muscle-fascia-fat-skin layers, ii) application of alloplastic materials as the meshes. A series of 124 immediate reconstructions were performed from 2008 to 2011 using a continuous composite pocket made of pectoralis maior and serratus anterior muscle above, and skin-fat flap below. The innovation is represented by an extended electrosurgical scoring of the lower pole of the mammary pocket at two levels. The first is the deep-fascia and muscle layer; the second is the superfiacial fascial system. This operative technique represents an advancement of a prior procedure described by the Authors in 1998. This preliminary study would primarily describe the technique step by step. Discussion debates about alternative techniques in terms of either surgical details of technique or cosmetic results are still to be reached.

\section{Introduction}

The cosmetic outcome following immediate breast reconstruction is generally improved by preservation of the native skin envelope. Toth and Lappert described skin-sparing mastectomy in 1991. ${ }^{1}$ The second and ultimate step was represented by preservation of nipple and areola as reconstructions without nipple are less pleasant. The nipple is the focal point of the breast and is frequently difficult to be optimally reconstructed. ${ }^{2}$

Based on both cosmetic inputs and conservative approaches to breast cancer, skin-sparing mastectomy (SSM) and nipple-sparing (NSM) types of mastectomy have found more indications in the treatment of the breast diseases, such as multifocal IDCS, T1-T2 IDC, T3 after tumor regression by neoadjuvant therapy. ${ }^{3}$ The risk of disease relapse is acceptable in patients with peripheral tumors smaller than 3 cm. ${ }^{4-7}$ In addition NSM is becoming the gold standard for any prophylactic treatment as risk-reducing mastectomy. ${ }^{8-10}$ Nevertheless preserving all skin envelope can represent a problem for both aesthetics and vascularity; some kind of skin-reducing mastectomy would be helpful in case of large ptotic breast. ${ }^{11}$

Starting from the 90 's, ${ }^{12}$ single-stage reconstruction by implants was consistently considered more complicated and risky. However extrusion, infection, and flap necrosis were reported in all types of immediate reconstruction. On the other hand, the worst outcomes obviously occurred if patient selection, improving surgical techniques, and choosing correct devices were not taken into account, as already suggested by Spear and Majidian in 1998. ${ }^{13}$ Other surgeons opted for permanent expander alone or immediately combined to latissimus dorsi flap. ${ }^{14}$ However the ideal solution is still far from being found.

In the meantime patient expectations for the best and most prompt cosmetic result have greatly increased compared to ten years ago. Nevertheless, even if the primary condition is given by preserving the nipple-areola complex (NAC) when oncologically safe, it is not sufficient to reach the real reconstructive goal. Sparing all the breast skin frequently results in a skin redundancy that is larger when the breast is big or hanging. The skin, after Cooper's ligaments resection, is free to extend its surface, especially if skin is less elastic (after pregnancy, weight loss or aging). Another side effect is represented by the risk for central skin necrosis. The latter seldom occurs after both correct patient selection and conservative dissection of the subcutaneous layer. Hence the real problem is to replace as much as possible the volume loss after parenchyma excision. The related drawbacks are skin folding, scar retraction, and NAC displacement that are very difficult to be corrected later and that dramatically damage breast aesthetics and body perception.

Then, from a reconstructive point of view, the best solution is provided by a rapid volume expansion capable of filling, or better overfilling, the residual skin envelope and stabilizing the nipple position in symmetric relation to the contralateral. This is attainable by the insertion of a definite high-cohesive silicone
Correspondence: Egidio Riggio, Plastic and Reconstructive Surgery Unit, Fondazione IRCCS Istituto Nazionale Tumori, via Venezian 1, 20133 Milano, Italy

Tel: +39.02.2390.2965 - Fax: +39.02.2390.2602.

E-mail: egidio.riggio@istitutotumori.mi.it

Key words: breast reconstruction, skin-sparing mastectomy.

Received for publication: 30 September 2011. Revision received: 12 December 2011. Accepted for publication: 19 December 2011.

This work is licensed under a Creative Commons Attribution NonCommercial 3.0 License (CC BYNC 3.0).

(C) Copyright E. Riggio et al., 2012

Licensee PAGEPress, Italy

Surgical Techniques Development 2012; 2:e3 doi:10.4081/std.2012.e3

implant instead of a saline expander totally inflated after 1-2 months. High-cohesive gel of the implant produces some strain strength on the envelope. This force is more stable compared to that present with saline solution or a less viscous gel, and can resist external tension force, e.g. the muscular contraction or scar-tissue retraction, with minor inner displacement. Bio-mechanics of the forces acting on a woman's breast and the physical properties of breast tissue are fundamental for any plastic surgery procedure of the breast but scientific data is poor in literature. ${ }^{15}$ The crucial question is anatomical because the definite prosthetic pocket must maintain the ideal characteristics: i) to be well vascularized, i.e. sub-muscular; ii) to be resilient, pliable, and adequately large; iii) to be separated from the subcutaneous mastectomy pocket and the axillary cavity, in order to lower the risk of seroma and infections; to be partially free of the pectoralis muscle contractions and stiffness but contemporarily avoiding any movement, malposition and malrotation of the implant.

The device pocket cannot be the same as the pocket prepared for an expander to be inflated progressively after surgery and then exchanged. In addition the pocket will be refined and improved at the second necessary operative time, while surgical refinements must be maximized in a single-stage reconstruction.

\section{Materials and Methods}

Since 1999 we were used to performing subcutaneous mastectomy releasing inferiorly all the muscular insertions (pectoralis maior). It was done together with the pectoralis fascia 
elongation above the serratus anterior, and rectus fascia if present, at the same level as the pre-existent inframammary fold. Selected breast was small to medium and the implant size was inferior to $300 \mathrm{~g}$. Silicone implants were pre-filled with cohesive gel, never using expandable devices like the Becker's or similar. They were few cases compared with the majority of two-stage reconstruction with temporary expansion and the results were quite unreliable and unpredictable.

Starting in 2008, the surgical technique was changed and improved introducing a modification based on the principles applied in our Institute, and published in 1998, for rebuilding the inframammary fold at the second stage of reconstruction after expander. ${ }^{16}$ The series consists of 105 patients. Mastectomy was unilateral in 86 cases and bilateral in 19. The register included 17 skin-sparing and 107 nipplesparing mastectomies for a total of 124 immediate reconstructions with permanent implant. All were anatomical devices style 410 except two small round devices (style 100 of 120 and $150 \mathrm{cc}$ ) manufactured by Allergan Inc. The smallest implant was ML170g; the biggest was FX560g. Twenty-one subjects underwent immediate contra-lateral augmentation. Contrary to the prior limited approach related to patients with smaller breast, this technique allows us to extend the one-stage reconstruction to patients with larger breast and even demanding total breast augmentation in $20 \%$ of cases.

\section{Preoperative selection and planning}

Patient selection is basic. The first approach concerns communication with the patient. Comprehension of plastic and cosmetic aspects is often obscured by the patient's troubles about recently discovered cancer. Frequently asked questions could be: silicone implant vs. autologous tissue; one-stage to two-stage reconstruction; expander vs. permanent implant; best shape vs. symmetry of the breasts; timing of possible contra-lateral surgery; preference for augmentation, mastopexy or reduction. The mood of many patients may not allow good understanding of all of these items together. In these subjects it is better to delay the choice for contra-lateral mammaplasty to a second stage regardless of the immediate implant insertion in the affected breast. The decision to plan a larger implant size and decide the ultimate augmentation of the healthy breast can be taken at a second stage. On the other hand, permanent implant can also be changed with another of better shape and volume corresponding to the contra-lateral breast at a second stage that becomes possible, but not necessary, if symmetry is already satisfactory following the previous immediate reconstruction.

Contemporary aesthetic surgery of the healthy breast is suggested in the majority of patients requiring total augmentation, whereas ptosis correction by pexy is better performed secondarily. Reaching acceptable symmetry is very difficult when either pexy or augmentation/pexy must match a reconstruction where the final breast shape and NAC position are to be verified after months. Once the NAC is advanced after mastopexy, it will be impossible to move it again to get symmetry with the spared NAC following mastectomy.

From a clinical point of view, exclusion criteria for the immediate reconstruction with the technique here described after conservative mastectomy are the following: i) large breast (D/DD breast cup size); ii) severe ptosis; iii) heavy smoker patient; iv) obese patient.

In choosing the incision, the breast size and degree of ptosis are important. The incision is usually lateral radial, beginning from just outside the areola (respectively at 3 o'clock for left and 9 for right breast) in nipple-sparing mastectomy, is slightly curved and extends for a variable distance depending on the breast size towards the axilla if necessary. This preference is explained by minor risk of dermal ischemia and by more accurate dissection around the nipple-areola complex. Other authors consider the radial incision, not only lateral but also vertical, to be reliable to prevent necrosis. ${ }^{17}$ Other incisions were applied to including prior lumpectomy scars. Areolar incision was partially performed when existing scars were present. Circum-areolar deepithelialization is rarely carried out.

Pre-op measurements depend on the anatomic landmarks of breast and chest wall and sizing of potential augmentation of the whole breast in the same way as one must check in aesthetic surgery. Width, height, and projection are to be measured choosing the type of implant. Bilateral reconstruction facilitates implant selection. Pre-op indications are seldom changed during the operation. Here width and height of implant are difficult to be planned exactly before the mastectomy compared to the choice of a temporary expander or to the second stage of expander substitution with implant. Intra-operatively soft tissue thicknesses must be checked verifying width and height of the device, and possibly modifying them by about $0.5-1 \mathrm{~cm}$ more or less. It is also useful to weigh the breast gland removed compared to the implant weight taking into account that is better to choose an implant a little bigger than the breast weight. Some soft tissue retraction and atrophy occur after healing or radiotherapy.

When contemporary enlargement of the contralateral breast is planned, augmentation is preferably sub-muscular with the aim of improving implant symmetry.

\section{Operative technique}

The first step is given by a surgical dissection that should spare the following anatomical levels: i) deep fascia overlying the lateral free border of the pectoralis maior; ii) deep fascia joining the lower insertions of the cited muscle and the serratus anterior (also described as superficial pectoral fascia) $;{ }^{18}$ iii) all the adipofascial frame of the infra-mammary fold (known as the infra-mammary superficial fascial system) (Figure 1). ${ }^{19}$

After the mastectomy time, the plastic surgeon must analyze: i) the quality of the anatomical layers (some conditions interfere with the pocket preparation, e.g. higher insertions of the pectoralis maior muscle far from the inframammary line); ii) the entirety of the prior mastectomy dissection plane along the pectoralis free border and along the inframmamary fascial system. Any leakage can be promptly sutured with vicryl $2 / 0$ but, if the musculofascial tissue begins to tear, the plan for permanent implant must be discontinued pro expander, contemporarily with moderate saline inflation.

After this checkup, the pocket dissection starts with the infero-lateral undermining of the serratus anterior fibers (Figure 2) and continues with the normal superior and inferomedial undermining of the pectoralis maior (Figure 3).

The pocket must fit the device base and the limits must coincide with the width and height of the chosen implant with the aim of reducing the risk of malposition and malrotation. The technique described here is contraindicated in very large and ptotic breast, which could be treated by special skin-reducing technique. ${ }^{11}$

Serratus muscle is split, if its thickness is not too thin, with the aim of leaving a layer of muscle fibers above the rib cage. There are several advantages: i) a lateral wall of the pocket making it more pliable; ii) some active work of the deeper part of muscle, which is preserved; iii) reduction of post-op pain.

The lower border of the pocket must reach the level of the inframammary fold in correspondence with the contralateral (Figure 4). Any downward over-dissection should be avoided. The fibromuscular tissue is to be partially scored medially, close to the sternal border, from the 4th rib down to the inframammary limit (Figure 5). Then, both the fibromuscular tissues and hereupon the proper deep fascia i.e. musculo-fascial, are to be totally released respectively, by electrocautery, along all the inferior fold under the pectoralis maior and serratus anterior as far as the most lateral portion of the submuscular pocket (Figure 6). The final maneuver gives access to the deep adipose layer infero-laterally where fat is generally thicker. The enlargement of the lower pocket is nearly $1-2 \mathrm{~cm}$.

The following step is fundamental and its 
fulfillment strictly depends on the preservation (or rebuilding, if possible) of the superficial fascial system together with either the deep or the superficial subcutaneous fatty layer. At the same level as the prior medial-to-lateral inframammary fold, the electrosurgical section must involve the superficial fascia (Scarpa's fascia at its bifurcation near the breast parenchyma) and related retinacula (connective frame also known as inframammary ligament) (Figure 7). All the scores are carried out through the previous release of deep fascia or likely just a few millimeters upwards, at the aim of not bottoming out the implant lower border. The total fascial release can finally produce an extra-coverage of the lower pocket from 3 to $5 \mathrm{~cm}$. wide, made of pliable soft tissue extended by the maneuver without interrupting the myofascial coverage. The anterior envelope of the device pocket becomes dual, i.e. composed of muscle layer at the upper two thirds, and skin-fat layer at the lower part. Soft tissues are free to better reshape the lower contour of the implant, without any musculofascial restraint. The most inferior part of the inframammary fascial system stays where it is. At the end of this maneuver the pocket should result at the same level as the planned inframmamary line but, if it is lower, a median anchorage of the inferior superficial fascia in the chest wall can be made by suturing with vicryl 0 in order to better define the external contour of the fold or to prevent any bottomingout (Figure 8).

The last step after implant insertion is the closure of the pocket access between the free border of the pectoralis maior and the surgical edge of the serratus anterior (Figure 9). The suture is carried out by figure-of-eight stitches of vicryl 2.0. Closure tension can be present related to volume and projection of the implant inside. There is some relation with width and

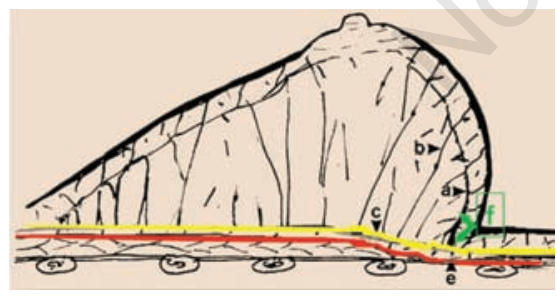

Figure 1. Breast anatomy related to the superficial fascial system shown in a sagittal section of the breast. (A) Fascia mammae showing the retinacula toward the skin, otherwise called infra-mammary ligament or the superficial layer of the fascia superficialis by different authors. (B) Cooper's ligaments. (C - yellow line) Fascia superficialis (Scarpa's superficial fascia). (Red line) Myofascial plane (deep fascia) showing short retinacula; E - level where the deep fascia is cut along the inframammary fold. (F) levels of the electrosurgical scoring of the superficial fascia and related retinacula. height. When devices are larger and tension occurs, muscular closure can be positively accomplished, unless deep fascia has not been excised together with breast parenchyma and hence muscle fibers tear off. Once suture is carried out, the residual strength on the muscle surface will be discharged after some week thanks to the biomechanical antagonism of the high coehesivity of the silicone gel. However, a trick suggested by the first author can facilitate the strain discharge. It consists of one or two sagittal partial scores into the fascia and uppermost fibers of the pectoral muscle. They are placed in the central thick part of muscle, 1-2 $\mathrm{cm}$ away from the suture line medially (Figure 10).

Breast implant is enclosed by an uninterrupted and well-vascularized, partially submuscular, pocket. The components are muscle, fas-
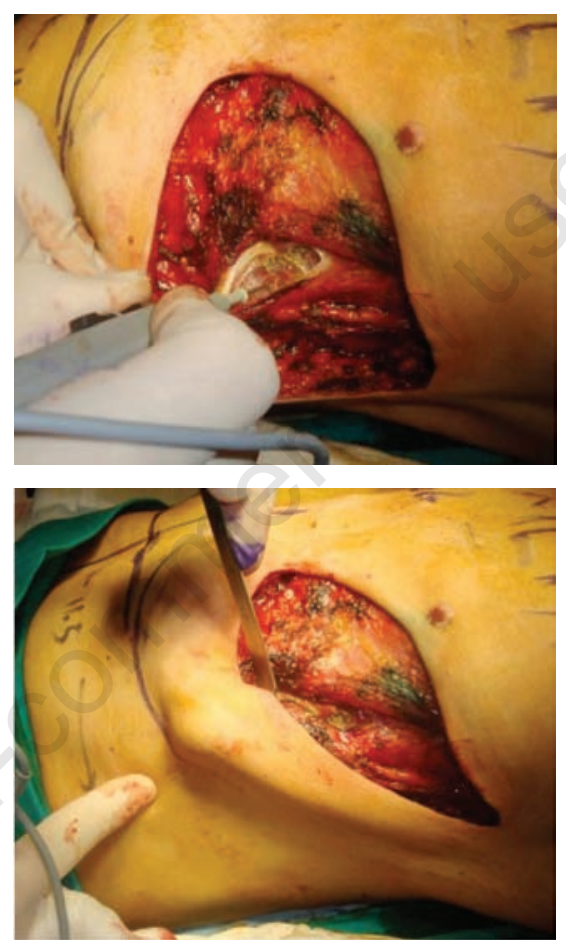

Figure 2. The pocket dissection: harvesting of the serratus anterior fibers infero-laterally.

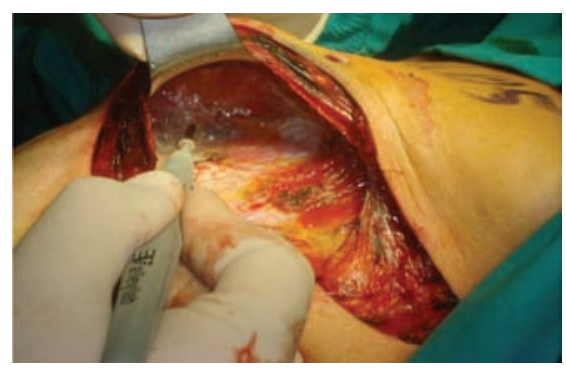

Figure 3. The pocket dissection: undermining of the pectoralis maior infero-medially and superiorly above the pectoralis minor muscle. cia, and skin-fat coming from the upper to the lower pole of the pocket. Hence, muscle action remains active in the upper half and implant can better expand the lower pole like a dualplane augmentation technique. There is a temporary compression of the implant projection, which gives some appearance of flattening and minor volume of the same implant (Figure 11).

\section{Discussion}

Three preliminary conditions must be present: i) the anatomical insertions of the pec-

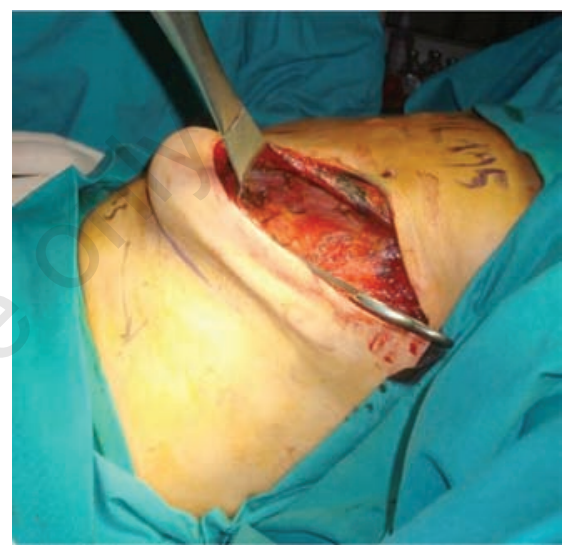

Figure 4. The lower border of the pocket reaches the inframammary fold line.
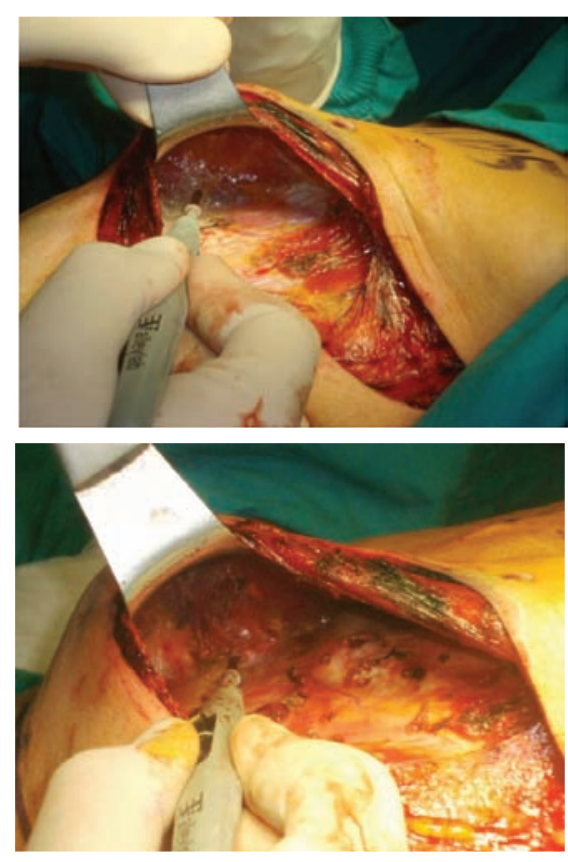

Figure 5. The fibromuscular tissue is partially scored medially, close to the sternal border, from the 4th rib down to the inframammary limit where a complete release starts. 

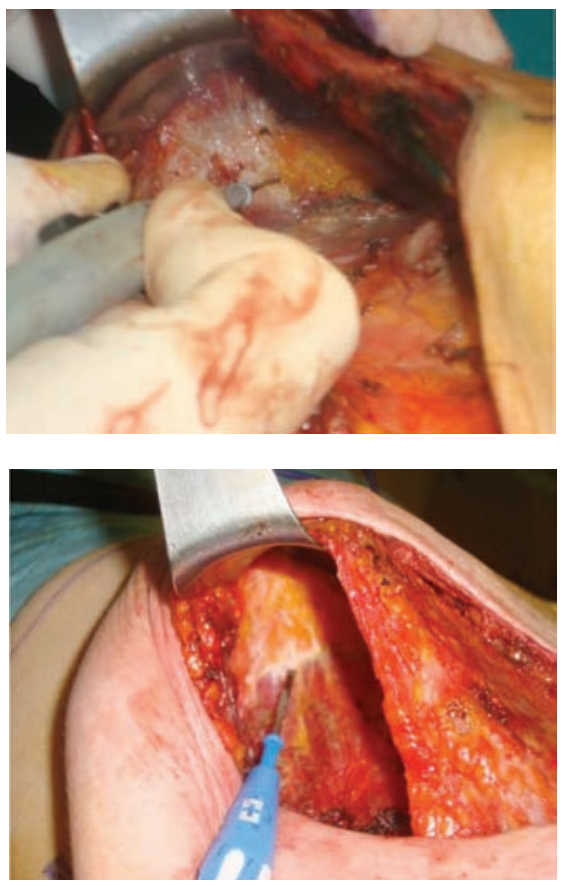

Figure 6. Both the fibromuscular tissues and the proper deep fascia. i.e. musculofascial, are totally released by electrocautery, along all the inferior fold under the pectoralis maior surface. This can produce a pocket extension of 1-2 cm.
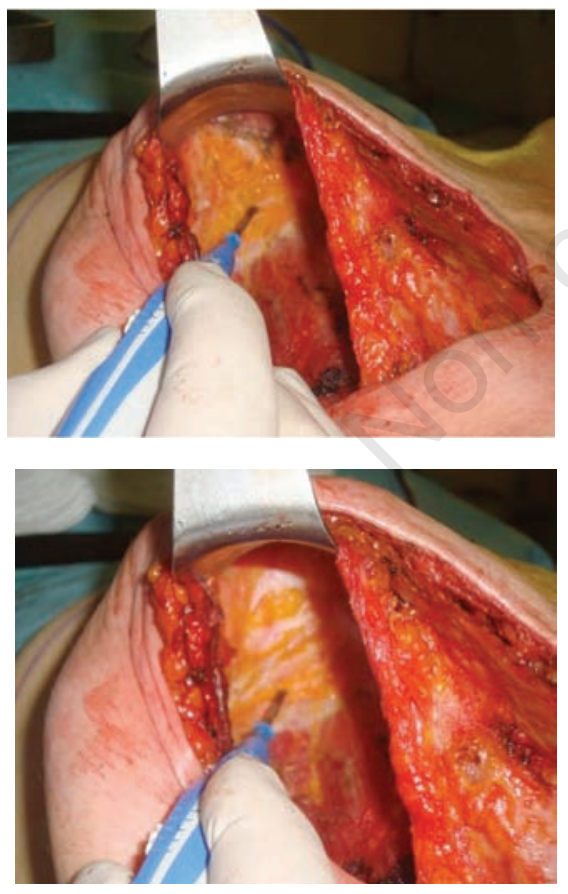

Figures 7. The electrosurgical section of the superficial fascia (Scarpa's fascia at its bifurcation close to the breast gland) and related retinacula (connective frame also known as inframammary ligament). Scoring the superficial fascia can give further extension of the pocket lower pole. toralis maior as close as possible to the inframammary fold; ii) the preservation of the deep fascia in all of the lower portion during mastectomy; iii) sparing all the subcutaneous layer along the inframammary fold (breast dissection is to be discontinued in front of the inframammary fascial system where glandular tissue is not present).

The implant is placed at the same level as the inframammary fold and symmetrical with the contralateral fold. Any lower dissection is unnecessary. The risk for upper displacement is greatly decreased by the wide muscular and fascial release at the lower pole. It is possible
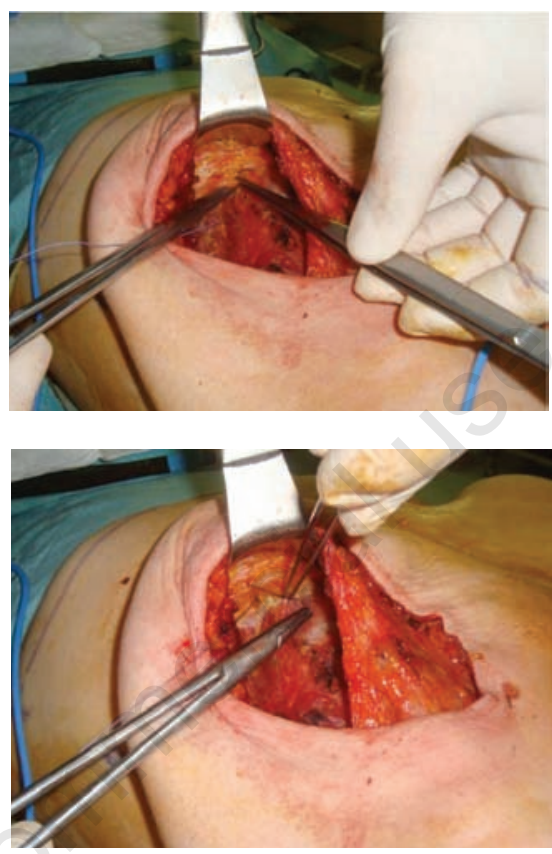

Figure 8. Median anchorage of the inferior edge of the superficial fascia is performed by suturing it to the deep plane with vicryl 0 stitches in order to better define the external contour of the fold or to prevent any bottoming-out.

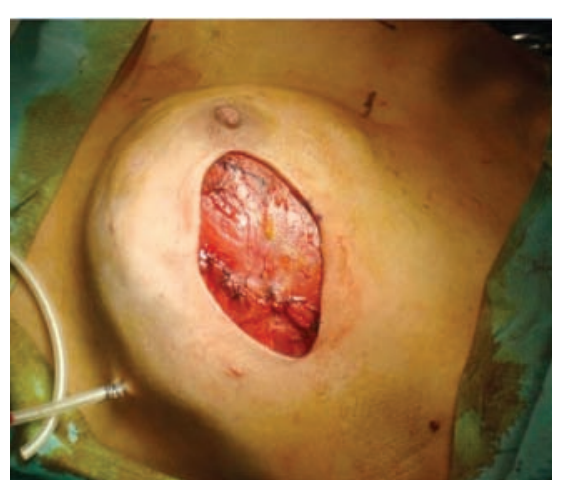

Figure 9. The pocket access is closed, after the implant insertion, with a vicryl 2.0 suture between the free border of the pectoralis maior and the surgical edge of the serratus anterior harvested. to dissect off the muscular fascia a few millimeters beneath the fold, but on the contrary the superficial fascia must be released a few millimeters above the fold perpendicularly in front of the skin as already described in part in the technique of inframammary fold reconstruction after expander substitution. ${ }^{16}$ We do not agree with other techniques that $a$ priori plan a lower malposition likely only to avoid another in the upper direction. ${ }^{20}$

As well, we cannot agree with plastic surgeons who plan to harvest the pectoral muscle destroying all fascial relationships at the submammary fold and rebuild a subcutaneous pocket using the lower mastectomy skin flap..$^{21}$ This maneuver involves progressive retraction of muscle fibres over time and potential complication risks in case of post-op breast irradiation.

Another focal point of the technique is the preservation of the lowest part of the breast soft tissue, which means not only in the inner plane but also in the outer, i.e. the skin. We have to disagree with all surgeons suggesting inframammary or vertical incision-access to the mastectomy. ${ }^{22}$ This maneuver can compromise any capability of natural expansion of the
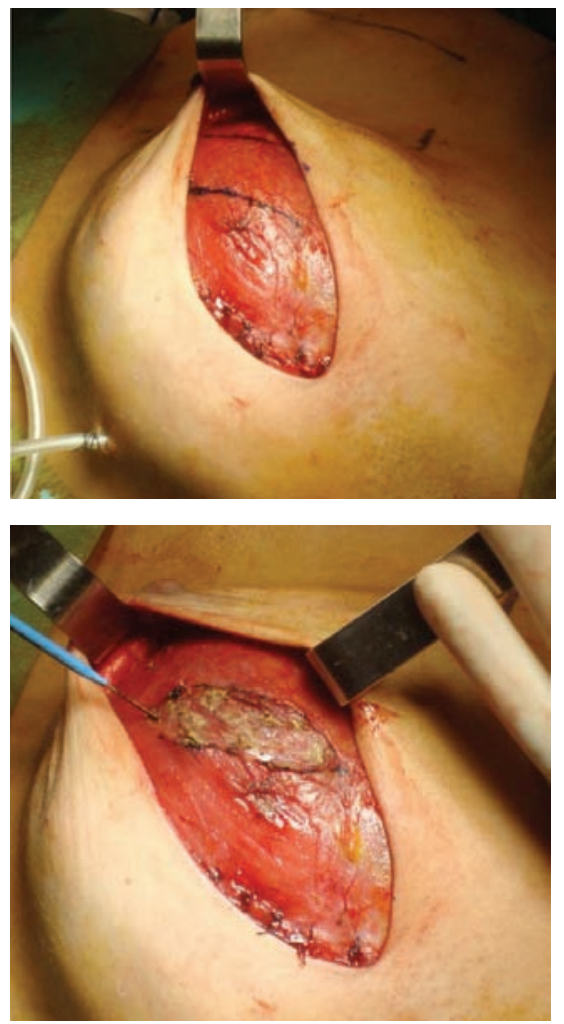

Figure 10 . One or two sagittal partial scores in the fascia and uppermost fibers of the pectoral muscle are sometimes performed to facilitate the strain discharge. They are placed in the middle thicker part of muscle, $1-2 \mathrm{~cm}$ far from the suture line medially. 


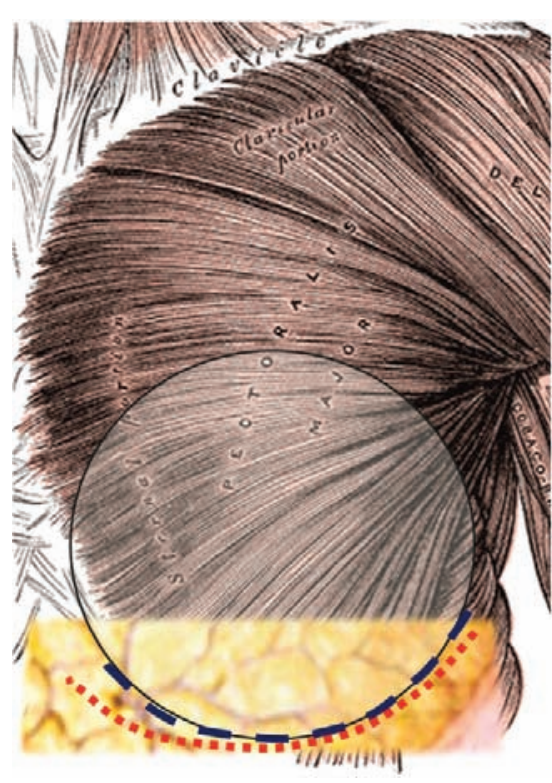

Figure 11. Composite pocket for immediate insertion of permanent silicone implant after mastectomy. The anterior part of the pocket is composed by pectoralis major (supero-medial) and serratus anterior (lateral) in the upper $2 / 3$ and by a continuous layer of soft tissue (fibroadipous layer and overlying skin) in the lower 1/3; the red dotted line indicates the section line of the muscles and deep fascia, exactly under the inframammary fold, the blue dotted line indicates the section line of the superficial fascial system a few millimeters above the previous incision. This is a retouched picture, i.e. digitally altered from its original version (M. pectoralis major by $H$. Gray, Anatomy of the Human Bod, Philadelphia: Lea \& Febiger, 1918). The original version can be viewed in Wikipedia: Gray410.png.

inframammary zone, and then necessitate an expander insertion instead of a definite prosthesis, or an alloplastic mesh reconstruction with major costs for the patient or the hospital. The overall preference for a lateral radial, even in presence of previous areola scars, is supported by data reported in literature and by our experience (less than $1 \%$ of areola necrosis, in this series). An incision of at least $30 \%$ of areola circumference was indicated as independent risk factor for necrosis by Garwood et al. decreasing the same to a $5 \%$ rate..$^{23}$ Of course, this can be insufficient when not associated to a dissection preserving the subcutaneous fatty layer and its vascular network. The same authors detected a further risk factor: the immediate reconstruction with fixed-volume using either implants or autologous tissue flap. They explained the current risk in autologous flaps because nipple-areola complex could survive better over a well vascularized pectoralis muscle during the first days. Based on biomechanical principles, it is not fixed-volume that represents a hazard, but the pressure

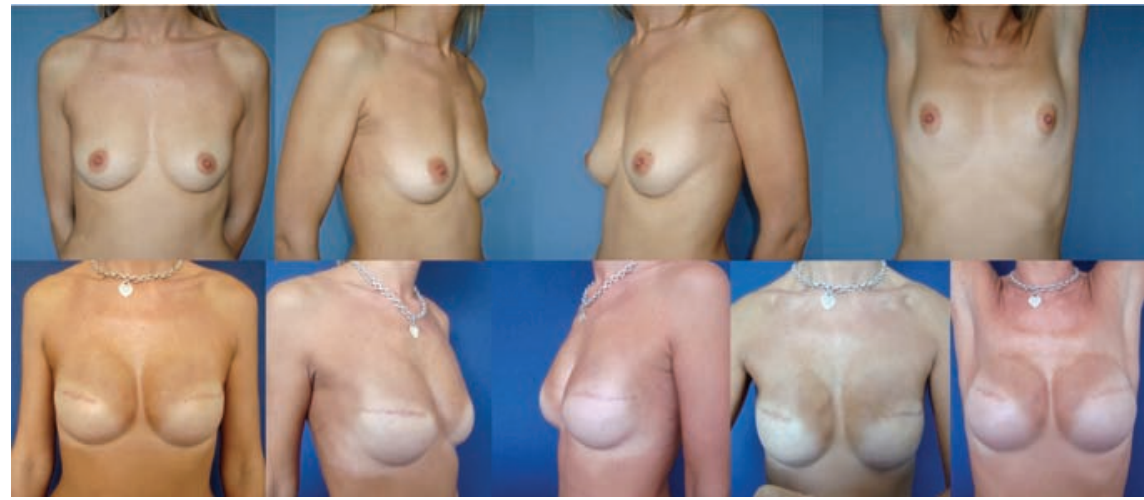

Figure 12. Bilateral skin-sparing mastectomy and immediate reconstruction with highcohesive gel anatomical implants with full-height/full-projection, (Allergan Natrelle 410FF 375g). Preoperative (above) and (below) postoperative patient's views.

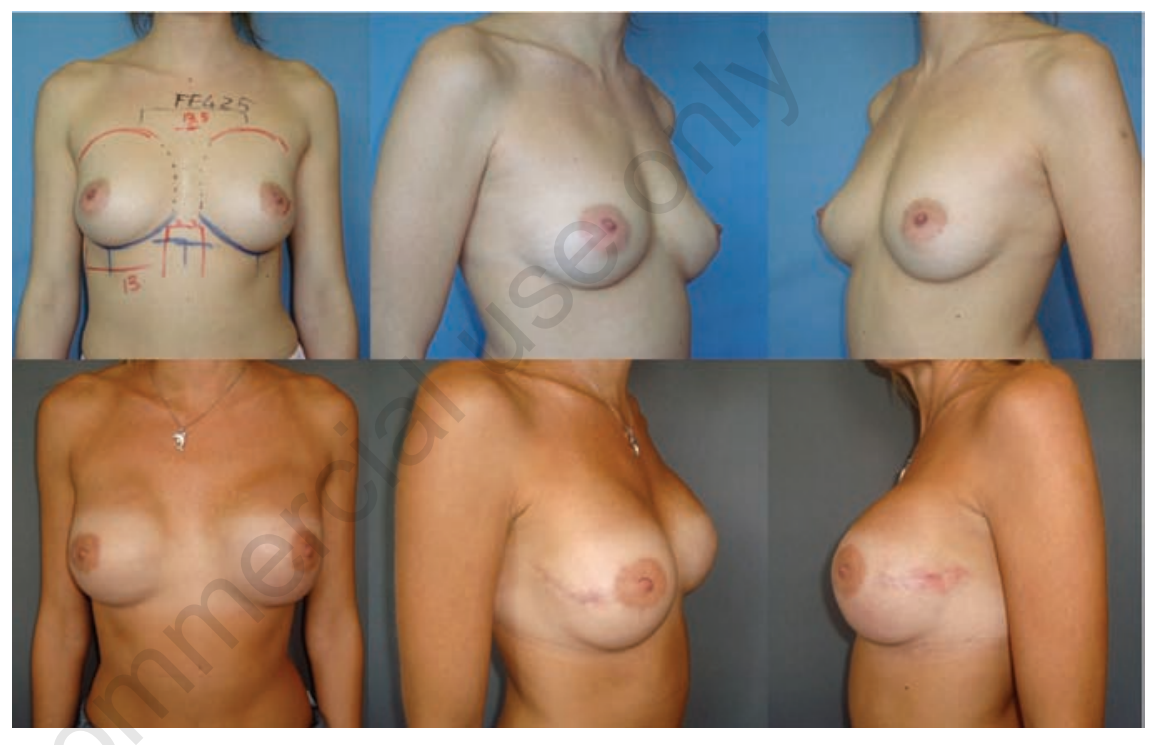

Figure 13. Bilateral nipple-sparing mastectomy and immediate reconstruction with highcohesive gel anatomical implants with full-height/full-projection, $425 \mathrm{~g}$ (Allergan Natrelle $410 \mathrm{FF} 425 \mathrm{~g}$ ). Preoperative (above) and (below) postoperative patient's views.

consistently generated on soft tissues. The pressure can result likely for a pendant autologous flap or for a big implant inserted in a subcutaneous pocket, whereas it seems to be provable for a complete muscular pocket where volume pressure is transmitted over the muscle fibers or against the chest wall instead of skin that is free to be draped comfortably.

When the study began, we prudently used implants which, on average, were less projected and big, whereas we are currently more confident with bigger size and projection by improving the fascial scoring technique (Figures 12 and 13), having observed the same device and soft tissue complications as occurring in reconstruction with temporary expanders contrary to other surgeon opinions. . $^{23,24}$

The immediate volume replacement does not produce any tension in skin flaps or add ischemic risk for nipple-areola survival. The related complications were not major compared to other types of mastectomy and reconstruction. Device pressure can be high depending on volume and projection but is totally discharged through the envelope of muscle fibers from the medial to lateral border of the pocket (pectoralis maior for nearly $60 \%$, and serratus anterior for the lateral part) against the chest wall during the first weeks. Moreover, skin surface after conservative mastectomy is generally loose and more capacious compared to the amount of breast parenchyma. Then skin envelope could often include a larger breast size, with poor tension. Immediate augmentation produces positive effects such as filling the skin envelope for reducing wrinkling, retraction, and areola-nipple malposition complication. Minimal or even major augmentation of the reconstructed breast is frequently suggested in our preop consultations. Major augmenta- 
tion was often associated with the contralateral at the same stage (in our series up to $25 \%$ of the subjects). Salgarello and Farallo performed contralateral augmentation surgery only in 11\% of the series, even though the implant was covered laterally by the muscular fascia, without serratus anterior muscle. ${ }^{20}$ The volume correction of minor differences were deliberately postponed after complete healing, i.e. at least six months later, and concerned $10 \%$ as minimal. The choice for a delayed operation of the healthy breast was prudently dictated by the most predictable evaluation of breast symmetry and shape. This became mandatory if the contralateral breast had need for some mastopexy with augmentation or alone.

We do not report any increased rate of revision operation compared to the large group of patients treated by expansion and two-stage reconstruction. When related to cancer histology, nipple-areola complex was later resected in local anesthesia. One implant partial extrusion after chemotherapy was solved by a skin surgical closure. There was no infection and no failure for implant removal. The well-vascularized pocket and the uninterrupted envelope above the implant are surely of great advantage compared to the reconstructive methods using alloplastic mesh (human and artificial acellular dermal matrix). On the contrary, these meshes present disadvantages in being very expensive and thin, and in becoming vascularized late.

Disadvantages of our technique are: i) strict selection of subject candidates at both the preoperative and intraoperative time and ii) greater skill required of the surgeon. Utilization of meshes could extend the indication to more breasts and subjects and does not require extra skill.

In conclusion, women's expectations for nipple-preserving mastectomy and satisfactory cosmetic results are demanding, and surgeons must be able to accomplish a single-stage reconstruction in as large a group of patients as possible improving the surgeon's skill and cosmetic attitude, reducing hospital admissions and costs, and contemporarily increasing patient satisfaction. Nevertheless, it is basic to inform the patient that: i) every immediate reconstruction can be considered as really definite in some cases but not all; ii) a secondary operation could always meliorate the prior result; iii) it is sometimes advisable to first carry out breast reconstruction with permanent implant in a single operation, and then delay the contralateral mammaplasty at a second operative time.

\section{References}

1. Toth BA, Lappert P. Modified skin incisions for mastectomy: the need for plastic surgical input in preoperative planning. Plast Reconstr Surg 1991;87:1048-53.

2. Wellisch DK, SchainWS, Noone RB, Little JWIII. The psychological contribution of nipple addition in breast reconstruction. Plast Reconstr Surg 1987;80:699-704.

3. Rusby JE, Smith BL, Gui GP. Nipple-sparing mastectomy. Br J Surg 2010;97:305-16.

4. Laronga C, Kemp B, Johnston D, et al. The incidence of occult nipple-areola complex involvement in breast cancer patients receiving a skin-sparing mastectomy. Ann Surg Oncol 1999;6:609-13.

5. Sacchini V, Pinotti JA, Barros AC, et al. Nipple-sparing mastectomy for breast cancer and risk reduction: oncologic or technical problem? J Am Coll Surg 2006;203: 70414.

6. Crowe JP, Patrick RJ, Yetman RJ, Djohan R. Nipple-sparing mastectomy update: one hundred forty-nine procedures and clinical outcomes. Arch Surg 2008;143:1106-10

7. Gerber B, Krause A, Dieterich M, et al. The oncological safety of skin sparing mastectomy with conservation of the nipple-areola complex and autologous reconstruction: an extended follow-up study. Ann Surg 2009;249:461-8.

8. Metcalfe KA, Semple JL, Narod SA. Time to reconsider subcutaneous mastectomy for breast-cancer prevention? Lancet Oncol 2005;6:431-4.

9. Garcia-Etienne CA, Borgen PI. Update on the indications for nipple-sparing mastectomy. J Support Oncol 2006;4:225-330.

10. Ashikari RH, Ashikari AY, Kelemen PR, Salzberg CA. Subcutaneous mastectomy and immediate reconstruction for prevention of breast cancer for high-risk patients. Breast Cancer 2008;15:185-91.

11. Nava MB, Cortinovis U, Ottolenghi J, et al. Skin-reducing mastectomy. Plast Reconstr Surg 2006;118:603-10; discussion 611-3.

12. Kroll SS, Baldwin B. A comparison of outcomes using three different methods of breast reconstruction. Plast Reconstr Surg
1992;90:455-62.

13. Spear SL, Majidian A. Immediate breast reconstruction in two stages using textured, integrated-valve tissue expanders and breast implants: A retrospective review of 171 consecutive breast reconstructions from 1989 to 1996. Plast Reconstr Surg 1998;101:53-63.

14. Gui GP, Tan SM, Faliakou EC, et al. Immediate breast reconstruction using biodimensional anatomical permanent expander implants: a prospective analysis of outcome and patient satisfaction. Plast Reconstr Surg 2003;111:125-38.

15. Gefen A, Dilmoney B. Mechanics of the normal woman's breast. Technol Health Care 2007;15;259-71.

16. Nava M, Quattrone P, Riggio E. Focus on the Breast Fascial System: A New Approach for Inframammary Fold Reconstruction. Plast Reconstr Surg 1998;102: 1034-45.

17. Stolier AJ, Sullivan SK, Dellacroce FJ. Technical considerations in nipple-sparing mastectomy: 82 consecutive cases without necrosis. Ann Surg Oncol 2008;15:1341-7.

18. Hwang K, Kim DJ. Anatomy of pectoral fascia in relation to subfascial mammary augmentation Ann Plast Surg 2005;55:5769.

19. Riggio E, Quattrone P, Nava M. Anatomical study of the breast superficial fascial system: the inframammary fold unit. Eur $\mathrm{J}$ Plast Surg 2000;23:310-5.

20. Salgarello M, Farallo E. Immediate breast reconstruction with definitive anatomical implants after skin-sparing mastectomy. Br J Plast Surg 2005;58:216-22.

21. Delgado JF, García-Guilarte RF, Palazuelo MR, et al. Immediate breast reconstruction with direct, anatomic, gel-cohesive, extraprojection prosthesis: 400 cases. Plast Reconstr Surg 2010;125:1599-605.

22. Maxwell GP, Storm-Dickerson T, Whitworth $\mathrm{P}$, et al. Advances in nipple-sparing mastectomy: oncological safety and incision selection. Aesth Surg J 2011;3 1:3109.

23. Garwood ER, Moore D, Ewing C, et al. Total skin-sparing mastectomy: complications and local recurrence rates in 2 cohorts of patients. Ann Surg 2009;249:26-32.

24. Wijayanayagam A, Kumar AS, Foster RD, et al. Optimizing the total skin-sparing mastectomy. Arch Surg 2008;143:38-45; discussion 45 . 